\title{
Approximating a solution to the two-part tariff problem
}

\author{
Ilko Vrankić ${ }^{1 * *}$, Mira $\operatorname{Krpan}^{1}$ and Zrinka Lukač ${ }^{1}$ \\ ${ }^{1}$ Faculty of Economics and Business, University of Zagreb \\ Trg J. F. Kennedy 6, 10000 Zagreb, Croatia \\ E-mail: 〈\{ivrankic, mkrpan, zlukac\}@efzg.hr〉
}

\begin{abstract}
The problem of setting the reservation price in terms of a two-part tariff requires, subject to new prices, reducing the difference between minimum expenditure for the starting level of utility and nominal consumer income. This difference in expenditure can be translated into the area below a compensated demand curve. The compensated demand curve is not directly observable, so the reservation price in this paper is approximated by a change in the consumer surplus. For the case of heterogeneous consumers, a number of reservation prices exist. This paper will address error estimation in setting prices of a capital good and a service. The results obtained are demonstrated using a numerical example.
\end{abstract}

Key words: two-part tariff, reservation price, heterogeneous consumers, error estimation

Received: October 6, 2014; accepted: February 2, 2015; available online: March 30, 2015

DOI: $10.17535 /$ crorr.2015.0011

\section{Introduction}

The two-part tariff is a pricing policy that enables a consumer to pay a fixed charge and a unit price [2]. It is common practice for paying utility bills, Polaroid cameras, amusement park fees, taxi charges and the like [9]. Pricing policies in amusement parks were the motivation behind Oi's article (1971) [6, 7].

This paper derives the solution algorithm for the well-known two-part tariff problem, formulated as a bilevel multi-follower program and approximated reservation price with the area left of the Marshallian demand curve and above the price line $[1,4,5]$. The upper level represents a monopolist who makes decisions about the price of capital goods and services with the goal of maximizing profit, whereas the lower level represents consumers divided into $n$ homogenous consumer groups who make independent decisions about their expenditure and quantity of goods consumed with the goal of maximizing utility.

${ }^{*}$ Corresponding author. 
In the two-part tariff problem, the consumer cannot enjoy the consumption of a service without the capital good, which has its price. Every consumer compares the fixed fee and the highest price he or she is willing to pay for the capital good. This highest price is called the reservation price and it is equal to the area left of the Hicksian or compensated demand curve and above the price line. They are unobservable in practice, as they require the utility to be held constant. Approximating the reservation price with the area left of the observable Marshallian or uncompensated demand curve and above the price line is useful. This area is called the consumer surplus. Existing literature usually starts from quasilinear preferences that neglect the income effect. This paper takes into account the income effect and the difference between uncompensated and compensated demand. In terms of representing consumer tastes, the CES utility function and error estimation are used to set the price of a capital good in the case of a consumer group.

\section{Model formulation}

To highlight fully the structure of the problem and the decision-making dynamics, the two-part tariff problem has been formulated as a bilevel multifollower programming problem [5]. In general, bilevel programming models are suitable for describing hierarchical decision-making situations. A decision maker at the top level - the leader - first makes a decision for a best outcome, i.e. maximizes or minimizes the objective. However, once the leader has made the decision, each of the decision makers at the subordinate level - the followers will make their own decisions independently, thus endeavoring to optimize the objective. The leader cannot force them to do whatever is best for the leader. Therefore, when making the decision, the leader is to anticipate the reactions of followers, given that their choices affect the feasible choice and overall outcome for the leader. The opposite is also true.

The two-part tariff problem is exactly this kind of problem. The decision maker is the monopolist at the top of the decision-making hierarchy, while the lower level represents consumers divided into homogenous groups, with each group characterized according to its income and substitution elasticity, and acts independently. The monopolist determines the prices of a capital good and a service, primarily to maximize profit. Once the prices have been determined, each consumer group independently decides whether to consume the good, and if so, how much of it. If the price of the capital good is less than or equal to the corresponding reservation price, a consumer group will consume the good and the level of demand is determined by solving a specific optimization problem, with the objective to maximize the group's utility. In return, each consumer group's choice affects the monopolist's overall profit. Therefore, when deciding prices, the monopolist has to consider each consumer group's reaction. 
In representing consumers' preferences, the starting point is the strongly increasing and strictly quasiconcave utility function, and the use of the composite commodity theorem to solve the following model of utility maximization subject to the given budget constraint [3,8],

$$
\begin{array}{r}
v(p, M)=\max _{x, m \geq 0} u(x, m) \\
p x+m=M .
\end{array}
$$

The solutions to the above optimization problem are the Marshallian demand functions, $x^{M}(p, M)$ and $m^{M}(p, M)$, and indirect utility function, $v(p, M)$. The service price $p$ and nominal consumer income determine the budget constraints.

Given that the consumer cannot enjoy the consumption of a service without the capital good which has its price, the dual problem of minimizing expenditure subject to the given utility level needs to be addressed, where the solutions are provided by the Hicksian demand functions $x^{H}(p, u)$ and $m^{H}(p, u)$ , and the expenditure function $e(p, u)$. Given that $e(p, u(0, M))$ are the minimum expenditures that ensure a level of utility where the consumer does not buy the capital good, the highest price the consumer is willing to pay for the capital good or the reservation price is equal to the difference between the nominal income and those expenditures [5],

$$
p^{r}=M-e(p, u(0, M))
$$

The CES utility function is chosen to represent consumer tastes. Let $n$ denote the number of consumer groups, $S_{i}$ the size of the consumer group $i, M_{i}$ the income of the consumer group $i, \sigma_{i}$ the substitution elasticity for the consumer group $i, c$ the marginal cost of a service that the capital good provides and let $\alpha$ be a sufficiently large number. The monopolist has to decide the price of the capital good, $p^{c}$, and the price of the service, $p$. The decision variables relating to each consumer from the consumer group $i$ consider the quantity of service consumed, $x_{i}$, expenditure on all other goods, $m_{i}$, and the reservation price, $p_{i}^{r}$. Moreover, they decide whether or not to consume the good, $\delta_{i}$, where $\delta_{i}=1$ if consumers from group $i$ are consuming the good in question and 0 if otherwise.

The problem can now be formulated in the form of the following bilevel multi-follower programming problem [5]: 


$$
\max _{p \geq 0, p^{c} \geq 0} \mathrm{~F}\left(p, p^{c}, \mathrm{x}, \mathrm{m}, \mathrm{p}^{\mathrm{r}}, \delta\right)=\sum_{i=1}^{n} s_{i} \cdot\left[p^{c}+(p-c) \cdot x_{i}\right] \cdot \delta_{i}
$$

s.t.

$$
\max _{x_{i}, m_{i}, p_{i}^{r}, \delta_{i}} U_{i}\left(x_{i}, m_{i}, p_{i}^{r}, \delta_{i}\right)=\left(x_{i}^{1-\frac{1}{\sigma_{i}}}+m_{i}^{1-\frac{1}{\sigma_{i}}}\right)^{\frac{\sigma_{i}}{\sigma_{i}-1}}
$$

s.t.

$$
\begin{aligned}
& p x_{i}+m_{i}=M_{i}-p^{c} \cdot \delta_{i} \\
& p_{i}^{r}=\left[1-\left(p^{1-\sigma_{i}}+1\right)^{\frac{1}{1-\sigma_{i}}}\right] \cdot M_{i} \\
& \delta_{i}= \begin{cases}1 ; & \text { if } p^{c} \leq p_{i}^{r} \\
0 ; & \text { otherwise }\end{cases} \\
& x_{i} \leq \alpha \cdot \delta_{i} \\
& x_{i}, m_{i} \geq 0, \delta_{i} \in\{0,1\}
\end{aligned}
$$

The monopolist determines the price of the capital good, $p^{c}$, and the price of the service, $p$, in order to maximize his profit function $F$, as described in (3). However, when making the decision, the monopolist has to take into account that each of the $n$ consumer groups will make a consumption decision independently based on their individual objectives and constraints, thus directly affecting the monopolist's profit. A consumer group will consume the good only if the price of the capital good is less than or equal to its reservation price. Therefore, the monopolist's profit comprises the sum of profits for each consumer group, where the profit for each consumer group $i$ is 0 if the group does not consume the good $\left(\delta_{i}=0\right)$, or $s_{i} \cdot\left[p^{c}+(p-c) \cdot x_{i}\right]$ if the group does consume the good. Hence, $\left[p^{c}+(p-c) \cdot x_{i}\right]$ is the profit for each group member and $s_{i}$ is the number of group members.

Once the monopolist has determined the price of the capital good and the price of the service, each of the $n$ consumer groups makes an autonomous decision on whether to consume that good while trying to maximize its utility (4). Therefore, the lower level consists of $n$ independent optimization subproblems, one for each consumer group. Since the groups are homogeneous, it suffices to consider the behavior of one individual consumer for each group. A member of a group $i$ has to decide whether to consume the good and the service offered by the monopolist, described by $\delta_{i}$, as well as the quantity of the service consumed, $x_{i}$, in order to maximize its utility $U_{i}$ as described by (4). The decision concerning the quantity of the service leads to a decision on the expenditure on other goods, $m_{i}$. The utility is maximized subject to a set of constraints (5) - (9). Should members of the group $i$ decide to buy the capital 
good, each of them has a remaining budget of $M_{i}-p^{c}$ to spend on buying the service $\left(p x_{i}\right)$ and other goods $\left(m_{i}\right)$, as shown by budget constraint (5). If the members of group $i$ are not buying the capital good, than $\delta_{i}=0$ and the entire budget is being spent on other goods. The reservation price for group $i$ is determined in (6). Members of group $i$ will buy the good and the service only if the price of the capital good is less than the corresponding reservation price, as shown in (7) and (8). All decision variables should be non-negative, as required by (9).

\section{Solution algorithm}

In general, bilevel programming problems are difficult to solve. Moreover, if the solution to the lower level problem is not unique, even the notion of a solution poses difficulties. Given the special properties of the two-tariff problem, the defined bilevel programming model for the problem in question will always have a unique solution. This section presents the algorithm for such a solution.

A brief overview of the problem is given here. A relationship exists between the price of the service, $p$, and the reservation price $p_{i}^{r}$ for each consumer group $i, i=1,2, \ldots, n$. Given the price of the service, $p$, the reservation price for a consumer group $i$ is uniquely determined by

$$
p_{i}^{r}=\left[1-\left(p^{1-\sigma_{i}}+1\right)^{\frac{1}{1-\sigma_{i}}}\right] \cdot M_{i} .
$$

Furthermore, if the price of the capital good $p^{c}$ is less than or equal to the reservation price $p_{i}^{r}$ of the consumer group $i$, consumers from group $i$ will consume the good $\left(\delta_{i}=1\right)$ and the quantity consumed will be determined by solving the optimization problem (4) - (9). For fixed $p, p^{c}$ and $\delta_{i}=1$, this problem has a unique solution which is analytically given by

$$
x_{i}=\frac{M_{i}-p^{c}}{p^{\sigma_{i}}\left(p^{1-\sigma_{i}}+1\right)} \text {. }
$$

In that case, expenditure on other goods is given by

$$
m_{i}=\frac{M_{i}-p^{c}}{p^{1-\sigma_{i}}+1} .
$$

If the price of the capital good is greater than the reservation price $p_{i}^{r}$ of the group $i$, consumers from that group will not consume the good $\left(\delta_{i}=0, x_{i}=0\right)$. They will spend their entire income on the other goods instead $\left(m_{i}=M_{i}\right)$.

The choice of the service price $p$ determines the reservation price for consumers from each of the consumer groups, which in combination with the 
price of the capital good, determines values of the decision variables for all consumer groups involved.

Consider the monopolist's objective function

$$
\mathrm{F}\left(p, p^{c}, \mathrm{x}, \mathrm{m}, \mathrm{p}^{\mathrm{r}}, \delta\right)=\sum_{i=1}^{n} s_{i} \cdot\left[p^{c}+(p-c) \cdot x_{i}\right] \cdot \delta_{i} \cdot
$$

Note that the monopolist's profit is not a continuous function. Since group $i$ consumes the good and therefore contributes to the profit only if its reservation price is less than or equal to the price of the capital good $p^{c}$, and the profit function may have discontinuities in all $p^{c}$ such that $p^{c}=p_{i}^{r}$ for $i=1,2, \ldots, n$.

Since a group's reservation price is a function of $p$, the domain of $p$ can be partitioned into intervals $I_{k}, j=1, \ldots, l$ such that $\bigcup_{k=1}^{l} I_{k}=[0,+\infty)$ with the property indicating that the groups' reservation prices maintain the same relationship to one another in terms of being less than or greater than one another. In other words, an interval $I_{k}$ is the maximum interval for which a permutation of group indexes $\Pi_{k}$ exists, such that

$$
p_{\pi_{k}(1)}^{r} \leq p_{\pi_{k}(2)}^{r} \leq \cdots \leq p_{\pi_{k}(n)}^{r} \quad \text { for all } p \in I_{k}
$$

Intervals $I_{k}$ are obtained by finding the intersections of $p_{i}^{r}$ and $p_{j}^{r}$ for all $i, j=1,2, \ldots, n, i \neq j$. If $0=p_{0}<p_{1}<p_{2}<\cdots<p_{l-1}$ are all the values of a service price for which some of the reservation prices are intersecting, then $I_{k}=\left[p_{k-1}, p_{k}\right], k=1, \ldots, l-1$ and $I_{l}=\left[p_{l},+\infty\right)$. Figure 1 provides an example of such intervals for two consumer groups.

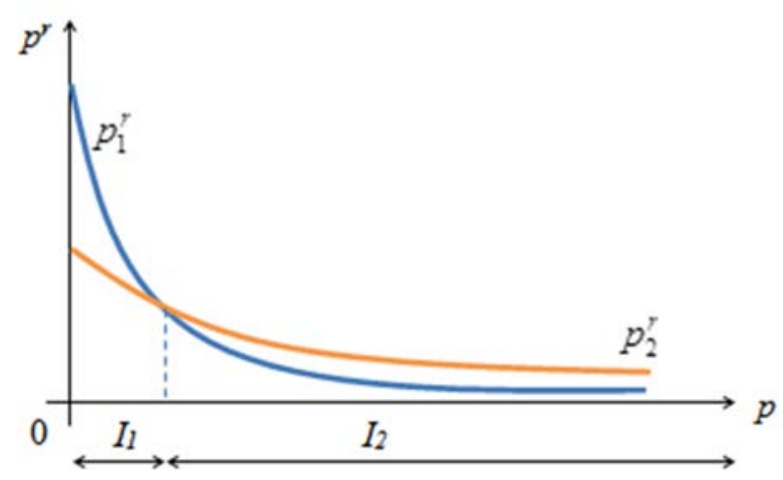

Figure 1: Reservation prices as functions of the service price $p p_{1}^{r} p_{2}^{r}$

For a given interval $I_{k}, k=1,2, \ldots, l$ and the corresponding permutation of the groups' indices $\Pi_{k}$, the value of $p^{c}$ will fall into some of the intervals $J_{t}^{k}$, 
$t=1,2, \ldots, n$, where $J_{t}^{k}=\left(p_{\pi_{k}(t-1)}^{r}, p_{\pi_{k}(t)}^{r}\right]$ with $p_{\pi_{k}(0)}^{r}=0$, or it will be $p^{c} \geq p_{\pi_{k}(n)}^{r}$. In case $p^{c} \geq p_{\pi_{k}(n)}^{r}$, it is true that

$$
p_{\pi_{k}(1)}^{r} \leq p_{\pi_{k}(2)}^{r} \leq \cdots \leq p_{\pi_{k}(n)}^{r} \leq p^{c},
$$

hence, none of the consumer groups consumes the good and the total profit is equal to 0 . If $p^{c} \in J_{t}^{k}, t=1,2, \ldots, n$, then

$$
p_{\pi_{k}(1)}^{r} \leq p_{\pi_{k}(2)}^{r} \leq \cdots \leq p_{\pi_{k}(t-1)}^{r} \leq p^{c} \leq p_{\pi_{t}(t)}^{r} \leq \cdots \leq p_{\pi_{k}(n)}^{r},
$$

hence, the profit function reduces to

$$
\mathrm{F}_{t}^{k}\left(p, p^{c}\right)=\sum_{i=t}^{n} s_{\pi_{k}(i)} \cdot\left[p^{c}+(p-c) \cdot \frac{M_{\pi_{k}(i)}-p^{c}}{p^{\sigma_{\pi_{k}(i)}}\left(p^{1-\sigma_{\pi_{k}(i)}}+1\right)}\right]
$$

where $\mathrm{F}_{\mathrm{t}}$ is a continuous function which increases depending on variable $p^{c}$. For a fixed $p$, its maximum is achieved for $p^{c}=p_{\pi_{k}(t)}^{r}$, where

$$
p_{\pi_{k}(t)}^{r}=\left[1-\left(p^{1-\sigma_{\pi_{k}(t)}}+1\right)^{\frac{1}{1-\sigma_{\pi_{k}(t)}}}\right] \cdot M_{\pi_{k}(t)} .
$$

Therefore, in order to find the maximum of the profit function (11) in interval $I_{k}$, it becomes necessary to compare maximums of the following singlevariable $n$ continuous functions

$$
\mathrm{F}_{t}^{k}(p)=\sum_{i=t}^{n} s_{\pi_{k}(i)} \cdot\left[p_{\pi_{k}(t)}^{r}+(p-c) \cdot \frac{M_{\pi_{k}(i)}-p_{\pi_{k}(t)}^{r}}{p^{\sigma_{\pi_{k}(i)}}\left(p^{1-\sigma_{\pi_{k}(i)}}+1\right)}\right], t=1,2, \ldots, n
$$

where $p_{\pi_{k}(t)}^{r}$ in interval $I_{k}$ is given by (15). The maximum of the profit function (11) in interval $I_{k}$ coincides with the maximum of the function $F_{t^{*}}^{k}$ such that

$$
\max _{p \in I_{k}} F_{\pi_{k}\left(t^{*}\right)}^{k}(p) \geq \max \left\{\max _{p \in I_{k}} F_{t}^{k}(p), t=1,2, \ldots, n\right\} .
$$

Therefore, the problem of finding the optimal service price in interval $I_{k}$ is reduced to solving $n$ well-known optimization problems of finding the singlevariable function maximum. The overall maximum of the profit function $F$ is the maximum over all intervals $I_{k}, k=1,2, \ldots, n$, that is

$$
\max _{p} F(p)=\max _{k=1, \ldots, l}\left\{\max _{p \in I_{k}} F_{t}^{k}(p), t=1,2, \ldots, n\right\} .
$$

By comparing the optimal values of all profit functions $F_{t^{*}}^{k}$ over all intervals $I_{k}$ the global optimum of the profit function $F$ is obtained. Consequently, the problem of finding the optimum of discontinuous function of several variables $F$ has been reduced simply to solving $k \cdot n$ single-variable optimization problems. 


\section{A numerical example}

Example 1: Consider a monopolist who has to determine the prices of a capital good and a service with respect to two homogeneous consumer groups, where the marginal cost of the service that the capital good provides is equal to 1 . Group 1 consists of 4 members, has an income of 15 and its substitution elasticity is equal to 2 . Group 2 consists of 2 members, where each member has an income of 40 and a substitution elasticity of 3 .

The monopolist has to determine the price of the capital good, $p^{c}$, and the service, $p$, in order to maximize its profit given by

$$
\max _{p \geq 0, p^{c} \geq 0} \mathrm{~F}\left(p, p^{c}, \mathrm{x}, \mathrm{m}, \mathrm{p}^{\mathrm{r}}, \delta\right)=4 \cdot\left[p^{c}+(p-1) \cdot x_{1}\right] \cdot \delta_{1}+2 \cdot\left[p^{c}+(p-1) \cdot x_{2}\right] \cdot \delta_{2} .
$$

Given the prices $p^{c}$ and $p$, each consumer from Group 1 will decide whether to consume the good offered by monopolist or not, and decide its quantity, by solving the optimization problem (4) - (9). The same is true for consumers from Group 2.

First, intervals $I_{k}$ are calculated. Figure 2 is a graph of reservation prices for Groups 1 and 2 as functions of $p$. They intersect for a service price of $p_{1} \approx 1.69$. Hence, there are two intervals, $I_{1}=[0,1.69]$ and $I_{2}=[1.69,+\infty)$. For $p \in I_{1}$ the following is true $p_{1}^{r}(p)<p_{2}^{r}(p)$ and therefore $\pi_{1}(1)=1$ and $\pi_{1}(2)=2$. For $p \in I_{2}$ the following is true $p_{2}^{r}(p)<p_{1}^{r}(p)$ and therefore $\pi_{2}(1)=2$ and $\pi_{2}(2)=1$.

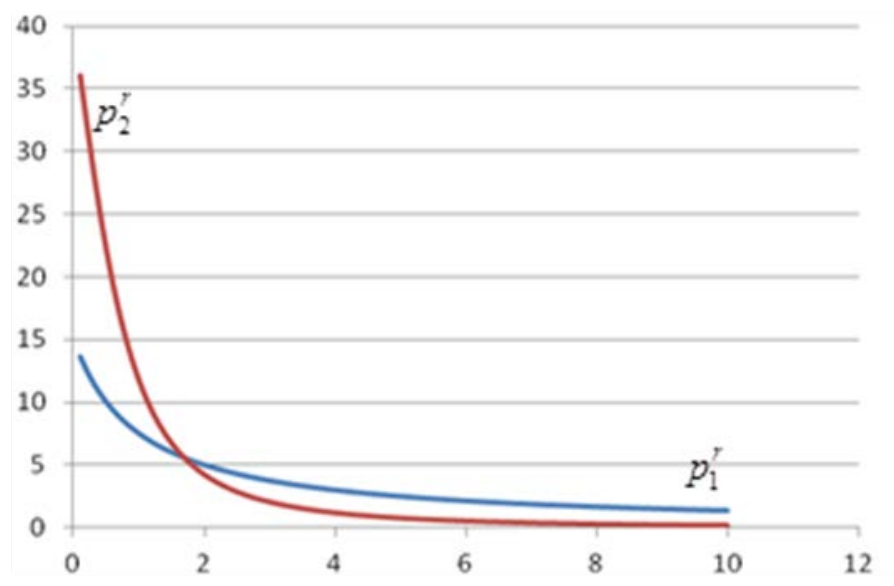

Figure 2: Reservation prices as functions of service price $p$

At interval $I_{1}$ the following partial profit functions $F_{1}^{1}$ and $F_{2}^{1}$ are considered, where 
Here

$$
\begin{gathered}
\mathrm{F}_{\pi_{1}(1)}^{1}(p)=\mathrm{F}_{1}^{1}(p)=4 \cdot\left[p_{1}^{r}+(p-1) \cdot \frac{15-p_{1}^{r}}{p^{2}\left(p^{-1}+1\right)}\right]+2 \cdot\left[p_{1}^{r}+(p-1) \cdot \frac{40-p_{1}^{r}}{p^{3}\left(p^{-2}+1\right)}\right] \\
\mathrm{F}_{\pi_{2}(2)}^{1}(p)=\mathrm{F}_{2}^{1}(p)=2 \cdot\left[p_{2}^{r}+(p-1) \cdot \frac{40-p_{2}^{r}}{p^{3}\left(p^{-2}+1\right)}\right] .
\end{gathered}
$$

$$
p_{1}^{r}=p_{\pi_{1}(1)}^{r}=\left[1-\left(p^{-1}+1\right)^{-1}\right] \cdot 15=\frac{15}{1+p} .
$$

At interval $I_{2}$ the following partial profit functions $F_{\pi_{2}(1)}^{2}$ and $F_{\pi_{2}(2)}^{2}$ are considered, where

$$
\begin{gathered}
\mathrm{F}_{\pi_{2}(1)}^{2}(p)=\mathrm{F}_{2}^{2}(p)=2 \cdot\left[p_{2}^{r}+(p-1) \cdot \frac{40-p_{2}^{r}}{p^{3}\left(p^{-2}+1\right)}\right]+4 \cdot\left[p_{2}^{r}+(p-1) \cdot \frac{15-p_{2}^{r}}{p^{2}\left(p^{-1}+1\right)}\right] \\
\mathrm{F}_{\pi_{2}(2)}^{2}(p)=\mathrm{F}_{1}^{2}(p)=4 \cdot\left[p_{1}^{r}+(p-1) \cdot \frac{15-p_{1}^{r}}{p^{2}\left(p^{-1}+1\right)}\right]
\end{gathered}
$$

with

$$
p_{2}^{r}=p_{\pi_{2}(1)}=\left[1-\left(p^{-2}+1\right)^{-\frac{1}{2}}\right] \cdot 40=40 \cdot\left(1-\sqrt{\frac{p^{2}}{1+p^{2}}}\right) .
$$

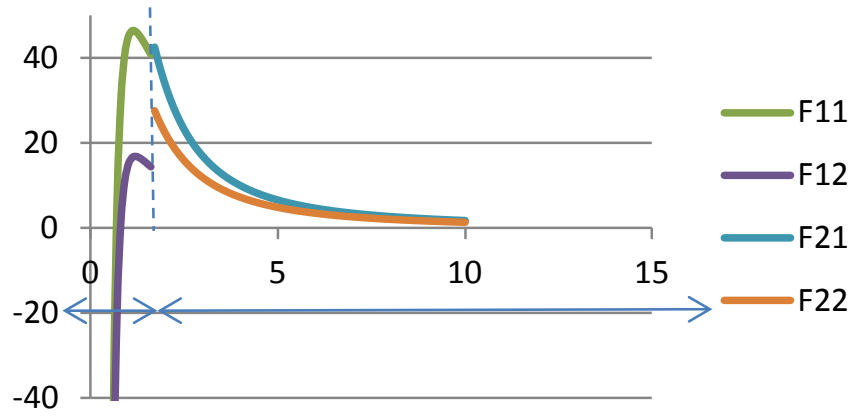

Figure 3: Partial profit functions as functions of service price $p$

Figure 3 shows the graphs of partial profit functions as functions of service price $p$.

The global maximum of the global profit function $F$ coincides with the maximum of the profit function $F_{1}^{1}$ in interval $I_{1}$ obtained for service price $p_{\max } \approx 1.324$ and is equal to $F^{\max } \approx 44.844$. The optimal price of the capital good is equal to the reservation price $p_{1}^{r}$, obtained for $p_{\max }$. Therefore, $p_{\max }^{c} \approx 6,45$. Both Groups 1 and 2 participate in the consummation of a good, Group 1 with respect to quantity $x_{1}^{\max } \approx 2.097$ and group 2 with respect to quantity $x_{2}^{\max } \approx 5.247$. 


\section{Approximating the solution of the two-part tariff problem and numerical example}

In maximizing profit, the monopolist has to take into account consumer reactions. If the price of the capital good is higher than the reservation price, the consumer will not enter the service market. In the previous section, the reservation price was presented as the difference between nominal income and minimal expenditure, ensuring a level of utility that corresponds to the situation where the consumer does not purchase the capital good. An analysis of the reservation price is given from another perspective and Figure 4 provides relevant information. Consider an initial point $\mathbf{x}^{\mathbf{0}}$ on an indifference curve $u(x, m)=u(0, M)$ encountering prices $p^{0}$ and a nominal income equal to the minimum expenditure, required to attain the utility level $u(0, M), e\left(p^{0}, u(0, M)\right.$. Now suppose that the service price decreases to $p^{1}$. The following question can be posed: how much better off is the consumer now that prices are lower? In other words, what is the maximum income a consumer is willing to pay at a lower price. This maximum is the amount that instills an indifference in the consumer with respect to the old and new situation [8]. This value is called the compensating variation and it is equal to the difference between the minimum expenditure required to attain the indifference curve $u(x, m)=u(0, M)$ at the new and old prices,

or, in the integral form

$$
C V=e\left(p^{1}, u(0, M)-e\left(p^{0}, u(0, M),\right.\right.
$$

$$
C V=\int_{p^{0}}^{p^{1}} \frac{d e(p, u(0, M))}{d p} d p .
$$

In line with Shephard's lemma, the expenditure difference for a small unit price increase is equal to the optimal quantity. Therefore, the compensating variation is equivalent to the area left of the Hicksian or compensated demand curve,

$$
C V=\int_{p^{0}}^{p^{1}} x^{H}(p, u(0, M)) d p .
$$

If the initial price is higher, the area under the Hicksian demand curve is greater and closer to the reservation price. The intercept on the vertical axis is then closer to $M$. Within limits, the area equals the reservation price,

$$
C V=\int_{p}^{+\infty} x^{H}(p, u(0, M)) d p .
$$

Given that the Hicksian demand curves are unobservable as they require the utility to be held constant, it is clear that the unobservable reservation price should be approximated to the area under the observable demand curves, i.e. the Marshallian demand curves or the uncompensated demand curves, 


$$
p^{r} \approx \int_{p}^{+\infty} x^{M}(p, e(p, u(0, M)) d p
$$

The approximation should not be greater than the reservation price, because the consumer group will not buy the good. Since the Marshallian demand curves require the income to be held constant, the only dilemma is selecting $M$ for errors to remain within reasonable boundaries.
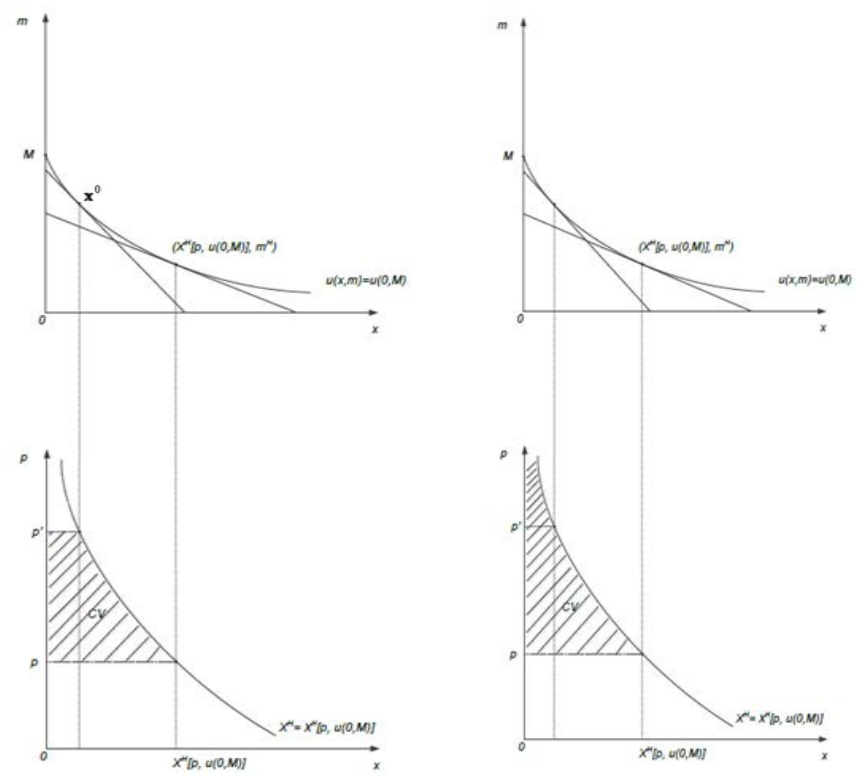

Figure 4: The compensating variation and the reservation price
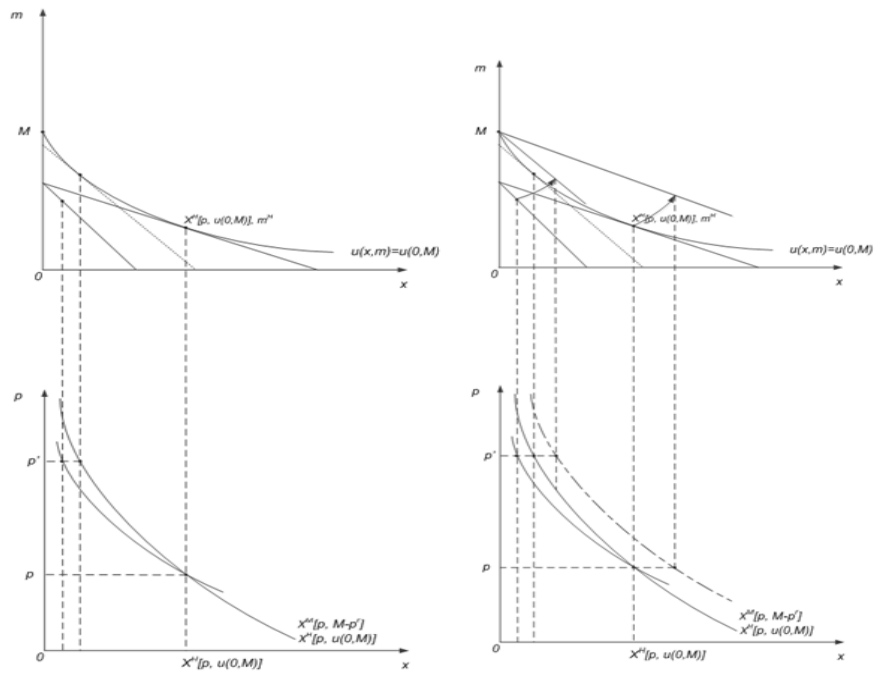

Figure 5: Hicksian and Marshallian demand curves 
If the expression $e(p, u(0, M)$ in the Marshallian demand function is replaced with $M$, the result is the demand curve illustrated in Figure 5 to the right. The goal is to reduce income as close as possible to the curve, $x^{H}$. In that case, there is a common point as illustrated in Figure 6.

The area under the resulting red curve can be divided into two parts. The area filled with the red slashes represents the area not contained in the approximation, whereas the area represented by the straight red lines represents the area contained in the approximation. Naturally, the goal is to reduce income so that these two areas are equal and thus the approximation becomes equivalent to the reservation price. Therefore, how much should income be reduced? Below is an approximation for only one consumer group. The assumption is that the marginal cost of a monopolist is constant and equal to 1 and that substitution elasticity for the consumer group is equal to 2 .

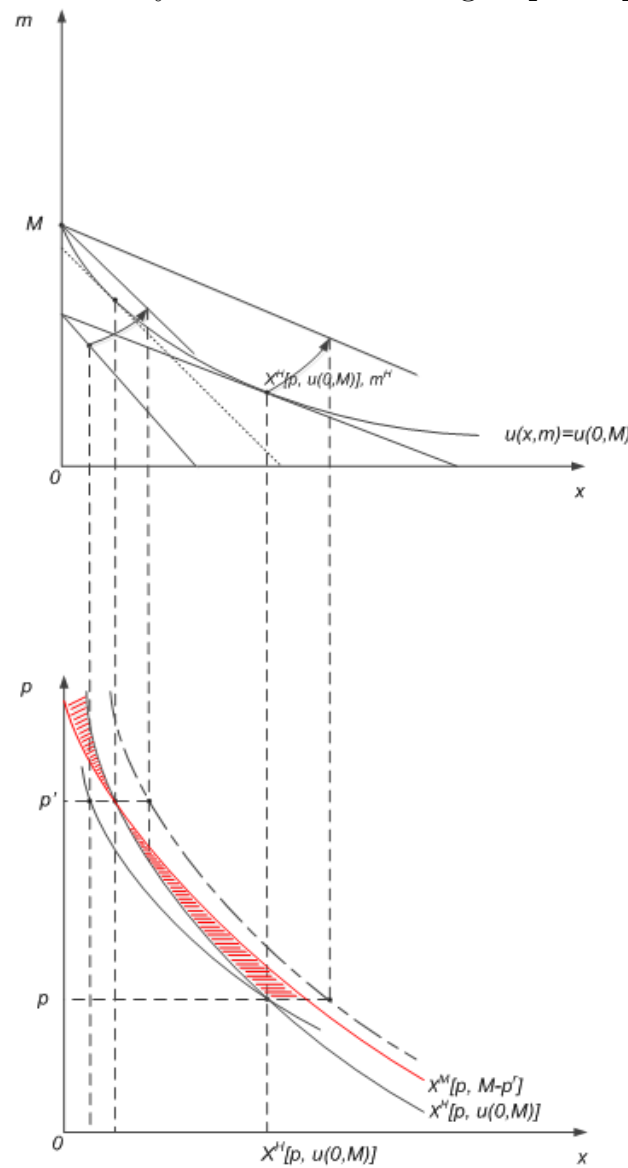

Figure 6: Approximating the reservation price to the area under the Marshallian demand curve 
In the case of one consumer group, as the service price is equal to the marginal cost, the area under the Marshallian demand curve for the CES utility function is given by the following integral

$$
\int_{1}^{+\infty} \frac{M}{p+p^{2}}=M \ln 2 .
$$

Table 1 below gives reservation price approximations for various income levels.

\begin{tabular}{|l|c|c|c|}
\hline M & 10 & 7 & 5 \\
\hline Japrox & 6.83 & 4.85 & 3.41 \\
\hline
\end{tabular}

Table 1: Approximating the reservation price to the area under the Marshallian demand curve

The true reservation price for the given parameters is equal to 5 . Subsequently, if the chosen income level is about 7, the error is small and difference between the monopolist's actual and maximum profit is small.

\section{Conclusion}

This paper has presented a construction of a solution algorithm for the wellknown two-part tariff problem. The algorithm was formulated as a bilevel multifollower program, which approximated the reservation price to the area left of the Marshallian demand curve and above the price line. The upper level consists of a monopolist who decides the price of a capital good and the price of a service with the goal of maximizing profit, whereas the lower level consists of consumers divided into $n$ homogenous consumer groups who autonomously decide the quantities of the good consumed in order to maximize their utility. If the price of the capital good is higher than the reservation price, the consumer will not enter the service market. Given that the reservation price is equal to the area left of the unobservable Hicksian demand curves and above the price line, this paper has sought to approximate it to the area left of the observable or Marshallian demand curves and above the price line, subject to conditions that make this approximation as accurate as possible. Existing literature usually starts from quasilinear preferences that neglect the income effect. This paper takes into account the income effect and the difference between uncompensated and compensated demand. The analysis precedes a numerical example for a consumer group whose tastes are represented by the CES utility function. Future research will focus more on the real assumption of the heterogeneity of clients with respect to taste and wealth. 


\section{References}

[1] Calvete, H.I. and Galé, C. (2007). Linear bilevel multi-follower programming with independent followers. Journal of Global Optimization, 39, 409-417. doi:10.1007/s10898-007-9144-2.

[2] DeSalvo, S. Joseph, and Huq, M. (2002). Introducing nonlinear pricing into consumer choice theory. The Journal of Economic Education, 33, 166-179. doi:10.1080/00220480209596465.

[3] Jehle, G. A. and Reny, P. J. (2001). Advanced Microeconomic Theory. Second Edition. Addison Wesley Longman.

[4] Lu, J., Shi, C. and Zhang, G. (2006). On bilevel multi-follower decision-making: General framework and solutions. Information Sciences, 176, 1607-1627. doi:10.1016/j.ins.2005.04.010.

[5] Vrankić, Ilko; Lukač, Zrinka; Oraić, Mira. (2011). Two-part tariffs and multistage programming. Croatian Operational Research Review (CRORR), 2, 232-242.

[6] Oi, Walter Y. (1971). Two-part tariffs for a Mickey Mouse monopoly. The Quarterly Journal of Economics, 85, 77-96. doi:10.2307/1881841.

[7] Schmalense, Richard (1981). Monopolistic two-part pricing arrangements. The Bell Journal of Economics, 12, 445-466. doi:10.2307/3003566.

[8] Silberberg, E. and Suen, W. (2000). The Structure of Economics: A Mathematical Analysis. Third Edition. Irwin/McGraw-Hill.

[9] Tirole, J. (1988). The Theory of Industrial Organization. Cambridge, MA: MIT Press. 